\title{
BMJ Open Rehabilitative management of back pain in children: protocol for a mixed studies systematic review
} Carol Cancelliere (1) , ${ }^{1,2}$ Jessica J Wong, ${ }^{1,2,3,4}$ Hainan Yu, ${ }^{1,2,4}$ Silvano Mior (D) ,
Ginny Brunton, ${ }^{1,5,6}$ Heather M Shearer, ${ }^{1,2,7}$ David Rudoler, ${ }^{1}$ Lise Hestbæk, ${ }^{8,9}$
Efrosini Papaconstantinou, ${ }^{1}$ Christine Cedraschi, ${ }^{10,11}$ Michael Swain, ${ }^{12}$
Gaelan Connell, ${ }^{1,2,13}$ Leslie Verville, ${ }^{1,2}$ Anne Taylor-Vaisey, ${ }^{2,4}$ Pierre Côté ${ }^{1,2,3,7}$

To cite: Cancelliere $\mathrm{C}$,

Wong JJ, Yu H, et al. Rehabilitative management of back pain in children: protocol for a mixed studies systematic review. BMJ Open 2020;10:e038534. doi:10.1136/ bmjopen-2020-038534

- Prepublication history and additional materials for this paper is available online. To view these files, please visit the journal online (http://dx.doi org/10.1136/bmjopen-2020038534).

Received 13 March 2020 Revised 03 September 2020 Accepted 08 September 2020

A) Check for updates

(C) Author(s) (or their employer(s)) 2020. Re-use permitted under CC BY-NC. No commercial re-use. See rights and permissions. Published by BMJ.

For numbered affiliations see end of article.

Correspondence to Dr Carol Cancelliere; carolina.cancelliere@ ontariotechu.ca

\section{ABSTRACT}

Introduction Little is known about effective, efficient and acceptable management of back pain in children. A comprehensive and updated evidence synthesis can help to inform clinical practice.

Objective To inform clinical practice, we aim to conduct a systematic review of the literature and synthesise the evidence regarding effective, cost-effective and safe rehabilitation interventions for children with back pain to improve their functioning and other health outcomes. Methods and analysis We will search MEDLINE, Embase, PsycINF0, CINAHL, the Index to Chiropractic Literature, the Cochrane Controlled Register of Trials and EconLit for primary studies published from inception in all languages. We will include quantitative studies (randomised controlled trials, cohort and case-control studies), qualitative studies, mixed-methods studies and full economic evaluations. To augment our search of the bibliographic electronic databases, we will search reference lists of included studies and relevant systematic reviews, the WHO International Clinical Trials Registry Platform and consult with content experts. We will assess the risk of bias using appropriate critical appraisal tools. We will extract data about study and participant characteristics, intervention type and comparators, context and setting, outcomes, themes and methodological quality assessment. We will use a sequential approach at the review level to integrate data from the quantitative, qualitative and economic evidence syntheses.

Ethics and dissemination Ethics approval is not required. We will disseminate findings through activities, including (1) presentations in national and international conferences; (2) meetings with national and international decision makers; (3) publications in peer-reviewed journals and (4) posts on organisational websites and social media. PROSPERO registration number CRD42019135009.

\section{INTRODUCTION}

\section{Rationale}

A significant proportion of children over 10 years of age suffer from back pain. ${ }^{1-5}$ The prevalence of back pain in children ranges between $4 \%$ and $74 \%$; the wide range is due to heterogeneous populations studied, outcome measurements and methodologies
Strengths and limitations of this study

- A systematic review integrating quantitative, qualitative and economic evidence to examine the rehabilitative management of back pain in children.

- Includes studies with a broad range of rehabilitation interventions as described by the WHO, and outcomes as described by the International Classification of Functioning, Disability and Health framework.

- Implements the Preferred Reporting Items for Systematic Review and Meta-Analysis Protocols guidelines.

There is no language restriction in articles.

- Our search strategies, while comprehensive, may miss relevant studies.

used. ${ }^{6}$ Data from the WHO Global Burden of Disease study in 2017 shows that low back pain is the leading cause of global years lived with disability. ${ }^{8}$ Back pain begins early in life with physical, mental and social consequences (eg, impact on school-related and sporting activities, general physical activity and wellbeing) that extend into adulthood. ${ }^{9-11}$ Most episodes of spinal pain are brief; however, in a 3-year prospective cohort study of 1465 school children in Denmark, up to $25 \%$ of children had three or more episodes over 1 year, and approximately $13 \%$ of children reported episodes lasting 5 or more weeks. ${ }^{12}$

Two recent systematic reviews assessed the effectiveness of manual therapy to treat a number of conditions including back pain in children, but low-quality evidence precludes drawing conclusions. ${ }^{13} 14$ A previous systematic review and meta-analysis which evaluated the effectiveness of conservative interventions for low back pain in children under 18 years of age reported that exercise interventions may be promising for improving pain scores in children compared with no 
treatment; however, the evidence was very limited and of low-quality. ${ }^{15}$ This evidence also needs updating. Additionally, to our knowledge, no integrative systematic review-one that incorporates both quantitative and qualitative studies-has been conducted regarding the rehabilitative management of back pain in children. Compared with traditional systematic reviews of quantitative studies, combining evidence of the effectiveness and efficiency of interventions with qualitative understanding from people's lived experiences can better inform clinical practice guidelines and policy. ${ }^{16}$

This comprehensive knowledge synthesis can inform clinical practice for decision makers involved with caring for children with back pain including healthcare professionals in a variety of clinical, rehabilitation or community settings (eg, physicians, nurses, physiotherapists, chiropractors, psychologists, occupational therapists, registered massage therapists). Moreover, the knowledge gaps that we identify can inform future research agendas.

\section{Objectives}

To support clinical practice for children with back pain, we aim to conduct an integrative systematic review of quantitative, qualitative and economic evidence regarding the rehabilitative management of back pain (including midback and low back pain) in children aged 19 years and under. Our review will address the following questions:

1 . What is the effectiveness and safety of rehabilitation interventions for improving functioning and other health outcomes in children with back pain?

2. What are the patients', caregivers' and providers' experiences, preferences, expectations and valued outcomes regarding rehabilitation interventions for back pain?

3. What is the cost-effectiveness of rehabilitation interventions for improving functioning and other health outcomes in children with back pain?

4. What can be hypothesised from the integration of the quantitative, qualitative and economic evidence about the effectiveness, cost-effectiveness and safety of rehabilitation interventions for low back pain in children?

We are targeting decision makers (clinicians, health managers/administrators, policy makers, patients and caregivers) involved in implementing, delivering or receiving rehabilitation interventions or programmes of care. We aim to provide them with knowledge regarding effective, acceptable and positively experienced interventions for children with back pain and their caregivers.

\section{METHODS}

We developed this systematic review protocol using the Preferred Reporting Items for Systematic Reviews and Meta-Analyses for Protocols (PRISMA-P) ${ }^{17}$ (see online supplemental additional file 1) and using methods already reported in detail elsewhere. ${ }^{18}$ We registered our protocol on the International Prospective Register of Systematic Reviews (PROSPERO) ${ }^{19}$ We will report our systematic review according to the PRISMA statement, ${ }^{20}$ and the Enhancing Transparency in Reporting the Synthesis of Qualitative Research reporting guideline. ${ }^{21}$

\section{Eligibility criteria}

Population

We will target studies including children (aged 19 years or younger) ${ }^{22}$ with non-specific low back or thoracic spine pain of any duration and severity. We define low back pain as pain and discomfort below the costal margin and above the inferior gluteal folds, with or without radiculopathy (referred leg pain). ${ }^{23}$ Radiculopathy refers to inflammation, injury/dysfunction or compression of spinal nerve roots that can present as pain, weakness or altered sensation in a myotomal or dermatomal distribution. Lumbar radiculopathy is commonly attributed to lumbar disc herniation (localised displacement of disc material beyond the normal margins of the intervertebral disc space) ${ }^{24}$ We define thoracic spine pain as pain within the region bounded superiorly by the first thoracic spinous process, inferiorly by the last thoracic spinous process and laterally by the most lateral margins of the erector spinae muscles. ${ }^{25}$ We will include studies investigating diagnoses including low back pain, mid-back pain, mechanical back pain, lumbago, lumbar sprain or strain, back sprain or strain, lumbopelvic pain, lumbar radiculopathy, lumbar disc herniation, sacroiliac syndrome, sciatica, dysplastic or isthmic spondylolisthesis or spondylolysis, musculoskeletal or non-specific chest wall pain (pain referred to the chest wall from the thoracic spine).

We will exclude studies of children with back pain attributed to major structural or systemic pathology (eg, fracture, acute traumatic or pathological spondylolisthesis or spondylolysis, infection, tumour, osteoporosis, inflammatory arthritides, cauda equina syndrome, neuromuscular disease, myelopathy and scoliosis); (2) studies of children with back pain attributed to a nonspine-related condition that might refer pain to the chest wall (eg, heart, lung or oesophagus conditions) and (3) studies that target asymptomatic children at baseline and assess interventions that aim to prevent the incidence of back pain.

\section{Intervention}

We will include studies that investigate the effectiveness and safety of rehabilitation interventions or programmes of care for children with back pain, including education and self-management strategies, exercise, manual therapies, passive physical modalities, acupuncture, pharmacological interventions, psychological interventions, environmental modifications, assistive devices and complementary therapies. Interventions may be delivered in any manner such as in-person, or remotely using technology such as telehealth. The WHO defines rehabilitation as a set of interventions that assist individuals who experience, or are likely to experience, disability to achieve and maintain optimal functioning when interacting with their environments (as described in detail 
previously). ${ }^{18} 26$ Rehabilitation interventions include rehabilitation medicine/therapy, which aims to: (1) improve function through the diagnosis and treatment of health conditions, reducing impairments, preventing or treating complications and (2) restore and compensate the loss of functioning, and prevent or slow deterioration in functioning in every area of a person's life. ${ }^{26}$ It may also include assistive devices, which refers to any item, piece of equipment or product used to increase, maintain or improve functional capabilities. ${ }^{26}$ Various healthcare providers may provide interventions including, but not limited to, general practitioners, nurses, physiotherapists, chiropractors, occupational therapists, psychologists and registered massage therapists (table 1$).{ }^{18} \mathrm{We}$ will exclude studies assessing surgical interventions, and interventions solely conducted at the societal level, such as barrier removal initiatives (eg, fitting a ramp to a public building).

\section{Comparison}

The quantitative component of this review will consider comparisons, including other interventions, placebo or sham interventions, wait list, standard care and no intervention.

\section{Outcomes}

Our primary outcome of interest is a child's functioning. Secondary health outcomes of interest are pain (eg, pain intensity, frequency or duration), psychological outcomes (eg, anxiety and depression), health-related quality of life, adverse events, qualitative outcomes and economic outcomes (table 2). We are interested in both short-term ( $<3$ months) and long-term ( $\geq 3$ months) outcomes. We selected these outcomes because they are important to children, their caregivers and decision makers and they are reflected in the WHO's framework for health and disability (International Classification of Functioning, Disability and Health (ICF)) (as described in detail previously). ${ }^{18} 27$ The ICF provides a standard language and framework for the description of health and health-related states, and organises information into two components-'body functions and body structures' and 'activities and participation'. ${ }^{27}$ Our primary outcome of interest, functioning, aligns with the 'activities and participation' component of the ICF. Examples of activities include walking, running, jumping and lifting. Participation refers to involvement in life situations such as with one's family, school and community. Common methods to measure functioning include the Modified Oswestry Low Back Pain Disability Questionnaire, ${ }^{28}$ Roland Morris Disability Questionnaire, ${ }^{29}$ return to school and participation in sports or other recreational activities. Pain and psychological outcomes fit within the 'body functions and body structures' component of the ICF. Common methods to measure pain include the Visual Analogue Scale (VAS),${ }^{30}$ Numerical Rating Scale (NRS), ${ }^{31}$ and Faces Pain Scale-Revised. ${ }^{32}{ }^{33}$ Common methods to measure psychological outcomes (eg, anxiety and depression) include
Revised Child Anxiety and Depression Scale ${ }^{34}$ and StateTrait Anxiety Inventory for Children. ${ }^{35}$ We will also assess health-related quality of life, which is not definable in the ICF framework. ${ }^{36}$ It is commonly measured with the KIDSCREEN-52, ${ }^{37}$ Pediatric Quality of Life Inventory ${ }^{38}$ and Patient-Reported Outcomes Measurement Information System Pediatric Self Report Scale. ${ }^{39}$ We defined adverse events or harms as any unfavourable sign, symptom, or disease temporarily associated with the treatment, whether or not caused by the treatment. ${ }^{40}{ }^{41}$ We will consider indirect harms (where the use of an intervention delays a diagnosis or treatment, and such delay holds a potential harm),${ }^{42}$ number of adverse events, severity of adverse events (ie, mild, moderate or severe) and number of participant withdrawals from the study due to adverse events. Qualitative outcomes include the experiences, preferences, expectations and valued outcomes (of children, caregivers and providers). Lastly, economic outcomes include direct costs (eg, resources saved by an intervention), indirect costs (eg, time freed by an intervention), economic health outcomes (eg, quality-adjusted life-year, incremental cost-effectiveness ratio, net monetary benefit) and intangible outcomes (eg, pain or suffering saved by an intervention).

\section{Types of studies}

We will include randomised controlled trials of any type (eg, superiority, non-inferiority and equivalence), cohort studies, case-control studies and mixed-methods studies (quantitative component) including any secondary analyses of eligible studies for question 1 (effectiveness and safety of interventions); qualitative and mixed-methods studies (qualitative component) for question 2 (users' experiences, preferences, expectations and valued outcomes of interventions) and trial-based and modelbased full economic evaluations for question 3 (cost-effectiveness of interventions) (table 2).

We will exclude the following types of studies: crosssectional studies, pilot studies assessing feasibility, protocol studies, case reports, case series, studies assessing only prevention of back pain and incidence outcomes, systematic reviews (although their reference lists will be searched for potentially relevant studies) and other review papers, clinical practice guidelines, biomechanical studies, laboratory studies, cadaveric or animal studies, conceptual papers, letters, editorials, commentaries, books and book chapters, conference proceedings, meeting abstracts, lectures and addresses, consensus development statements, guideline statements and studies reviewing solely partial economic evaluations (eg, cost of illness studies).

\section{Context and setting}

We will consider rehabilitation interventions/programmes of care delivered in any healthcare system within an urban or rural area and in any healthcare setting (eg, acute care, hospital, primary healthcare, rehabilitation clinics), or in the community (as described in detail previously). ${ }^{18}$ Community-based rehabilitation is implemented through 
Table 1 Examples of rehabilitation interventions

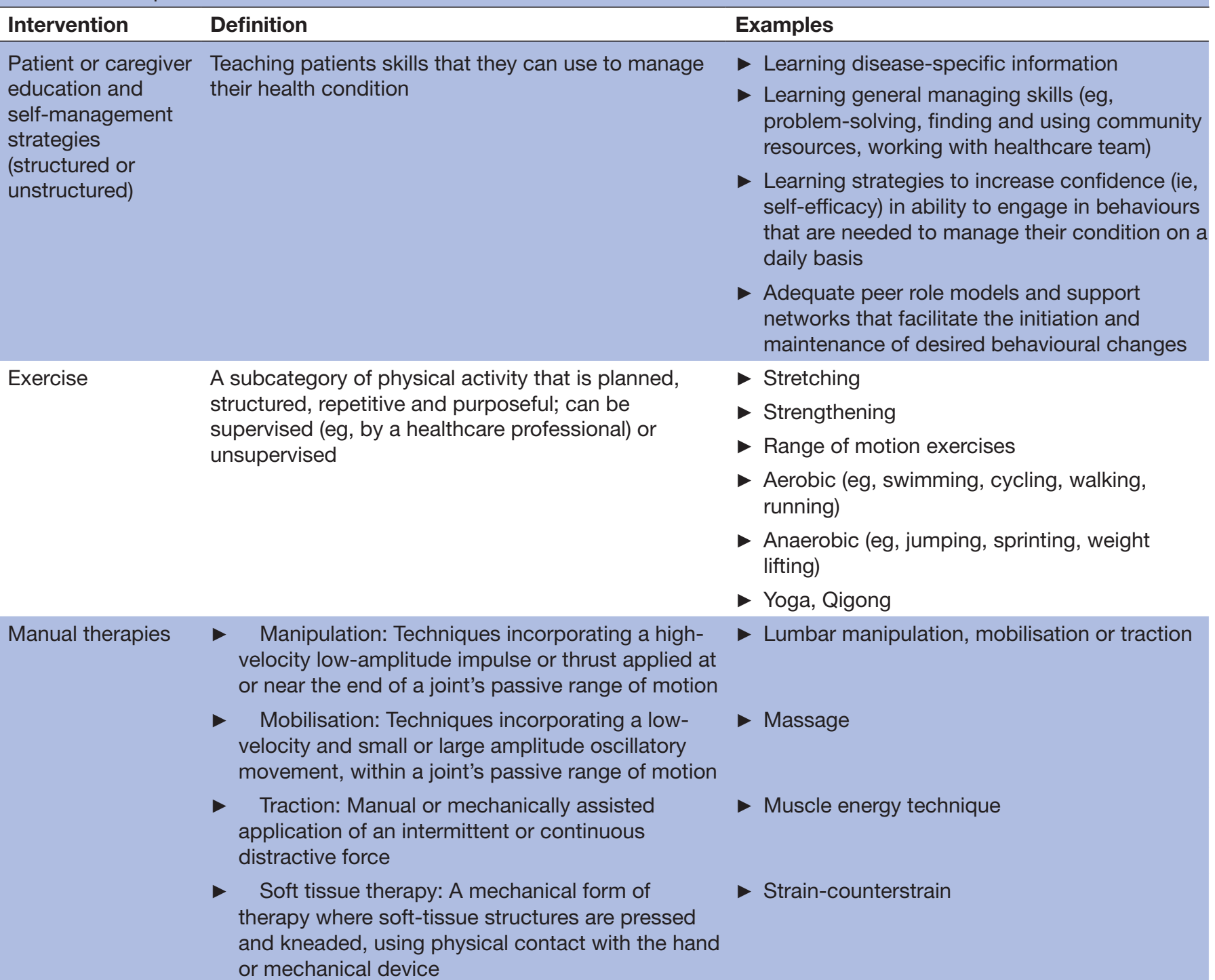

Passive physical modalities
A form of cold, heat or light application affecting the body at the skin level or ultrasonic or electromagnetic radiation affecting structures beneath the skin surface:

- Passive assistive devices: Device to encourage immobilisation in anatomic positions or actively inhibit or prevent movement
Heat application: heat pack, hydrotherapy

Cryotherapy: cold pack, vapocoolant spray

Low-level laser

- Electrical muscle stimulation

- Pulsed electromagnetic therapy

- Traditional needling

- Dry needling

- Burning of specific herbs

- Electro-acupuncture

- Photo-acupuncture

- Acetaminophen

- Nonsteroidal anti-inflammatory drugs

- Muscle relaxants

- Antidepressants 
Table 1 Continued

\begin{tabular}{|c|c|c|}
\hline Intervention & Definition & Examples \\
\hline $\begin{array}{l}\text { Psychological } \\
\text { interventions }\end{array}$ & $\begin{array}{l}\text { Activities used to modify behaviour, emotional state or } \\
\text { feelings }\end{array}$ & $\begin{array}{l}\text { Cognitive behavioural therapy } \\
\text { Counselling } \\
\text { Social network and environment-based } \\
\text { therapies } \\
\text { Psychoeducational interventions } \\
\text { Mindfulness meditation }\end{array}$ \\
\hline Assistive devices & $\begin{array}{l}\text { Any item, piece of equipment or product system, } \\
\text { used to increase, maintain or improve the functional } \\
\text { capabilities of people with disabilities }\end{array}$ & $\begin{array}{l}\text { Walking aids } \\
\text { Orthoses } \\
\text { Braces } \\
\text { Wheelchairs }\end{array}$ \\
\hline
\end{tabular}

the combined efforts of individuals with disabilities, their families and communities and relevant government and non-government health, education, social and other services (eg, advocacy programme). ${ }^{43}$

\section{Information sources}

We will develop the initial search strategy in MEDLINE, in consultation with an experienced health sciences librarian. A second experienced health sciences librarian will review the search strategy assessing its appropriateness and comprehensiveness using the Peer Review of Electronic Search Strategies Checklist. ${ }^{44}$ We will conduct electronic searches of the following databases from database inception to the present: MEDLINE (Ovid), Embase (Ovid), PsycINFO (Ovid), CINAHL (Cumulative Index to Nursing and Allied Health Literature, EBSCOhost), the Index to Chiropractic Literature (Chiropractic Library Collaboration), the Cochrane Controlled Register of Trials (Ovid) and EconLit (EBSCOhost). We will augment our search of the bibliographic electronic databases to identify additional relevant studies, and mitigate the potential impact of publication bias and selective outcome reporting bias. ${ }^{46}$ We will search reference lists of included studies from the database searches and relevant systematic reviews; and we will consult with content experts. We will ask experts to suggest up to three targeted websites that may contain relevant studies and other potentially relevant studies not captured by our search strategy. Lastly, we will search the WHO International Clinical Trials Registry Platform (http://apps.who.int/trialsearch/). For studies only reported in the registry, we will contact first authors by email (with two reminders over 1 month) to obtain full study reports, or additional study or outcome data. We will include studies in any language and will use professional medical translation services where required. If 12 or more months elapse between the search date and submission for publication, we will update the search.

\section{Search strategy}

The searches will include a combination of subject headings specific to databases (eg, MeSH in MEDLINE) and free text words to capture the key concepts of rehabilitative management of back pain in children (see online supplemental additional file 2).

\section{Patient and public involvement}

Patients were not involved in the design of our study. However, we will seek patient and public consultation during the development of clinical practice guidelines, which will be the next phase of this project.

\section{Data management}

We will download the electronic search results into EndNote V.X9 reference manager software (Clarivate Analytics, Pennsylvania, USA). We will remove duplicates and upload the remaining references to the Evidence for Policy and Practice Information and Coordinating (EPPI) Centre Reviewer software for the data extraction stages (EPPI-Reviewer V.4, UCL Institute of Education, University of London, UK). EPPI-Reviewer software stores references, manages and monitors the data extraction process and provides an audit trail for the review. ${ }^{47}$

\section{Screening for eligibility}

Using the inclusion and exclusion criteria, pairs of reviewers will independently screen titles and abstracts, and subsequently the full text of each selected article in order to confirm inclusion into the study (as described in 
Table 2 Research questions, outcomes and study types

$\begin{array}{lll}\text { Research question Outcomes } & \text { Study types }\end{array}$

What is the effectiveness and safety of rehabilitation interventions for improving functioning and other health outcomes in children with back pain?

Primary

1. Functioning: for example, Modified Oswestry Low

Back Pain Disability Questionnaire, Roland Morris Disability Questionnaire, return to school, participation in sports/other recreational activities

\section{Secondary \\ Cohort studies}

2. Pain (including pain intensity, frequency, duration): for Case-control studies example, VAS, NRS, Faces Pain Scale-Revised

3. Psychological outcomes (including anxiety and depression): for example, Revised Child Anxiety and Depression Scale, State-Trait Anxiety Inventory for Children

4. Health-related quality of life: for example, KIDSCREEN-52, Pediatric Quality of Life Inventory, PROMIS Pediatric Self Report Scale

5. Adverse events: any unfavourable sign, symptom, or disease temporarily associated with treatment, indirect harms (eg, delayed diagnosis/treatment), number of adverse events, severity of adverse events (ie, mild, moderate, severe), number of participant withdrawals from study due to adverse events.

What are the patients', caregivers' and providers' experiences, preferences, expectations and valued outcomes regarding rehabilitation interventions for back pain?

What is the cost-effectiveness of rehabilitation interventions for improving functioning and other health outcomes in children with back pain?

\section{Qualitative outcomes: experiences, preferences, expectations, valued outcomes}

Mixed-methods studies (quantitative component)

\section{Direct costs: resources consumed or saved by an intervention \\ Indirect costs: productivity gains or losses (eg, time consumed or freed by the intervention) \\ Economic health outcomes: QALY, ICER, NMB \\ Intangible: for example, pain or suffering saved or brought on by an intervention}

Qualitative studies (eg, phenomenology, grounded theory, ethnography, action research, descriptive qualitative studies)

Mixed-methods studies (qualitative component)

Full economic evaluations (trial-based and modelbased): cost-effectiveness, cost-utility, cost-benefit, costconsequences

ICER, incremental cost-effectiveness ratio; NMB, measure of net monetary benefit; NRS, Numerical Rating Scale; PROMIS, patient-reported outcomes measurement information system; QALY, quality adjusted life years; VAS, Visual Analogue Scale.

detail previously). ${ }^{18}$ Titles and abstracts will be classified as possibly relevant or irrelevant. Subsequently, full-text articles of abstracts classified as possibly relevant will be retrieved, reviewed and classified as relevant or irrelevant.

We will conduct training exercises prior to initiating the screening process to ensure reliability between reviewers. Reviewers will first screen a random sample of 50 records based on titles and abstracts. Paired reviewers must reach $90 \%$ agreement before completing title and abstract screening for the remaining studies. ${ }^{48}$ If this threshold is not reached for all review teams, all team members will discuss differences in classification to clarify and potentially modify the eligibility criteria prior to completing title and abstract screening. Next, reviewers will screen a random sample of 25 full-text articles. All paired reviewers must again reach $90 \%$ agreement before completing full-text article screening for the remaining studies. If not, all team members will discuss to clarify eligibility criteria and resolve disagreements prior to completing full-text article screening. On completing full-text article screening, paired reviewers will discuss disagreements and reach consensus related 
to the inclusion of any article, involving a third reviewer if necessary.

\section{Risk of bias in individual studies}

We will critically appraise studies according to study design using appropriate checklists (see online supplemental additional file 3) (as described in detail previously). ${ }^{18}$ We will assess the quality of studies using the Scottish Intercollegiate Guidelines Network (SIGN) criteria for randomised controlled trials (RCTs), cohort and case-control studies ${ }^{49}$; the Joanna Briggs Institute (JBI) Critical Appraisal Checklist for qualitative studies ${ }^{50}$; the Mixed Methods Appraisal Tool (MMAT) for mixed-methods studies $^{51}$ and the Drummond checklist for economic evaluations. ${ }^{52}$ The SIGN checklists allow reviewers to assess internal validity by considering the impact of selection bias, information bias and confounding on study results. The JBI checklist allows reviewers to assess the possibility of bias in qualitative studies' design, conduct and analysis. The MMAT allows reviewers to assess the interdependent qualitative and quantitative components of the study and criteria to consider, such as justification for mixing evidence, and appropriate ways of integrating the data. The Drummond checklist allows reviewers to identify elements that demonstrate a sound economic evaluation such as the assessment of both costs and effects of interventions, accurate measurements of costs and effects and allowances made for uncertainty in the estimates of costs and effects. We will contact the authors of papers to request missing or additional data for clarification where required. Paired reviewers will independently assess the eligible studies for quality. Any disagreements that arise between the reviewers will be resolved through discussion, or with a third reviewer. Since some of the reviewers have published within this area, the review coordinator will ensure that reviewers will not be assigned their own studies for risk of bias assessment. Additionally, reviewers will recluse themselves from any discussion and decisionmaking that involves their paper. We will clearly describe this in our final systematic review report.

Using these established checklists and notes to guide our assessment, we will categorise the validity or credibility of each study as either high, low, or unclear risk of bias. We will not use a quantitative cut-off score to determine study quality and will not pre-define weights for the checklist items. Rather, we will make an overall quality judgement by considering the impact of selection bias, information bias and confounding on study results throughout the conduct of each study. ${ }^{53}$ We will report detailed results of the critical appraisal in a narrative form and in a 'risk of bias' table. All studies, regardless of their methodological quality, will be extracted and synthesised (where possible). The overall methodological quality of relevant studies will be considered in the individual synthesis of quantitative, qualitative and economic data and the integration of these findings. The results of the risk of bias assessment will be used in a sensitivity analysis to ensure that studies judged to be at 'high risk of bias' do not affect the robustness of our results.

\section{Data items and data extraction process}

Paired reviewers will independently extract the data from all eligible studies. For the quantitative studies, we will extract data on the study and participant characteristics; intervention and comparator intervention characteristics using the Template for Intervention Description and Replication (TIDieR) checklist ${ }^{54}$; all pre-determined outcomes including multiple measures if used; key findings; and methodological quality. The TIDieR checklist ${ }^{54}$ consists of items to help readers better understand the interventions and how they were delivered (ie, name of intervention, why, what (materials), what (procedure), who provided, how, where, when and how much, tailoring, modifications, how well (planned), how well (actual) ). ${ }^{54}$ We will use the PerSPecTIF question formulation framework to guide data extraction for the qualitative studies regarding the items: perspective, setting, phenomenon of interest, environment, timing and findings (eg, themes). ${ }^{55}$ We will also extract data describing the qualitative approach used and methodological quality of studies. For both quantitative and qualitative studies, we will extract data on the ICF categories 'environmental factors' (contextual factors that make up the physical, social and attitudinal environment in which people live and conduct their lives) and 'personal factors' (internal contextual factors that influence how disability is experienced by the individual) to add context to the interventions and outcomes. ${ }^{27}$ For the economic evaluations, we will use the Consolidated Health Economic Evaluation Reporting Standards statement ${ }^{56}$ and extract data on the analytic approach (trial-based or model-based), evaluation type, the analytic perspective, time horizon adopted for costs, main cost items, setting, key findings and methodological quality of studies.

Paired reviewers will pretest the data extraction form and revise as needed. We will use EPPI-Reviewer software to manage the data extraction process. Any disagreements that arise between the reviewers will be resolved through discussion, or with a third reviewer. We will contact authors of papers to request missing or additional data, if required.

\section{DATA SYNTHESIS}

We will use a sequential approach at the review level to synthesise and integrate the data (as described in detail previously). ${ }^{18} 57$ This will involve separate quantitative, economic and qualitative findings synthesis followed by integration of the resultant quantitative, economic and qualitative evidence.

\section{Quantitative synthesis}

We will stratify studies to conduct separate comparisons according to study design, population, intervention, comparison, outcome and methodological quality and 


\section{Table 3 Categories to guide the analysis (meta-analysis or qualitative synthesis)}

\section{Study design Primary synthesis: \\ Design: RCTs vs non-RCTs (ie, cohort, case- control) \\ Subgroup analysis: \\ Specific RCT: for example, superiority, non- inferiority or equivalence

$\begin{array}{ll}\text { Population } & \text { Primary synthesis: } \\ & \text { Pain duration: acute/subacute pain (ie, }<12 \text { weeks' } \\ & \text { duration) vs persistent pain ( } \geq 12 \text { weeks' duration) } \\ & \text { Age range: infants (aged }<1 \text { year), children (aged } \\ & \text { 1-9years), or adolescents (aged 10-19years) } \\ & \text { Type of back pain: thoracic spine pain with/ } \\ & \text { without radiculopathy, low back pain with/without } \\ & \text { radiculopathy, musculoskeletal chest wall pain, } \\ & \text { spondylolisthesis/spondylolysis } \\ & \text { Subgroup analysis: } \\ & \text { Pain severity: mild, moderate or severe } \\ \text { Intervention } & \text { Primary synthesis: }\end{array}$ \\ Intervention type: education/self-management strategies, manual therapy, passive physical modalities, acupuncture, pharmacological intervention, psychological intervention, multimodal care, environmental modifications, assistive devices and complementary medicine \\ Subgroup analysis: \\ Specific intervention type: for example, type of exercise (eg, stretching vs aerobic) and type of manual therapy (eg, mobilisation, manipulation, traction, soft tissue therapy)}

$\begin{array}{ll}\text { Comparison } & \text { Primary synthesis: } \\ & \begin{array}{l}\text { Comparator type: active (other intervention) vs } \\ \text { inactive (eg, placebo/sham intervention, wait list, } \\ \text { standard or usual care, or no intervention) }\end{array} \\ \text { Outcome } & \text { Primary synthesis: }\end{array}$

Outcome type: functioning (eg, ODI, RMDQ), pain (eg, VAS, NRS), psychological (eg, Revised Child Anxiety and Depression Scale), health-related quality of life (eg, KIDSCREEN-52), or adverse events (eg, number, severity)

Time of outcome assessment: short-term ( $<3$ months) or long-term ( $\geq 3$ months)

Type of effect estimate:* for example, mean difference, relative risk, OR, or HR

$\begin{array}{ll}\text { Methodological } & \text { Primary synthesis: } \\ \text { quality } & \begin{array}{l}\text { Methodological quality assessment: low or unclear } \\ \text { risk of bias }\end{array} \\ & \begin{array}{l}\text { Sensitivity analysis: low, unclear and high risk of } \\ \text { bias }\end{array}\end{array}$

*If data are unavailable to re-express effect estimates into a common effect estimate (if applicable).

NRS, Numerical Rating Scale; RCTs, randomised controlled trials; RMDQ, Roland Morris Disability Questionnaire; VAS, Visual Analogue Scale.

further conduct subgroup analyses within categories (table 3). Specifically, we will stratify results to conduct separate comparisons between RCTs versus non-RCTs targeting, for example, children with acute low back pain without radiculopathy, treated with a specific intervention (eg, manual therapy) compared with an active comparison, and assessed by the mean difference in functioning score (eg, Oswestry Disability Index score) at 3 months.

We will assess clinical, methodological and statistical heterogeneity among studies (as described in detail previously). ${ }^{18}$ Differences in populations, interventions, comparators or outcomes across studies may result in clinical heterogeneity. Methodological and statistical heterogeneity may result from differences in risk of bias and differences in outcomes across studies beyond what could be expected by chance alone. We will assess the methodological heterogeneity across studies using our assessments from the SIGN checklist as either high, low, or unclear risk of bias. We will assess statistical heterogeneity using the $I^{2}$ statistic, whereby $I^{2}$ of $<25 \%-50 \%$ will be considered low to moderate (homogeneous), and $\geq 50 \%$ considered high (heterogeneous) ${ }^{58}$ If two or more studies are clinically homogeneous (ie, similar populations, interventions, comparators and outcomes) and statistically homogeneous (ie, $I^{2}<25 \%-50 \%$ ), we will perform a random effects meta-analysis using EPPI-Reviewer software using the relative risk (or OR for rare events) effect measure for dichotomous data, mean differences for continuous data, hazard rate ratios for time-to-event data and rates or rate ratios for count data. For studies that used multiple measures to assess the same outcome and at multiple time points, we will select the most prevalent measure and time point used across the studies to maximise the comparability of the findings. We will attempt to summarise the results in a similar way if possible. We will contact study investigators to obtain the data if it is not reported. If the data are unavailable, we will summarise the data in three ways: by entering the means as continuous outcomes, the counts as dichotomous outcomes and by entering all of the data in text form as 'other data' outcomes. ${ }^{59}$ We may also use statistical approaches to re-express ORs as standardised mean differences (and vice versa), allowing us to combine dichotomous and continuous data. ${ }^{59}$ For our primary analysis, we will analyse the studies with low and unclear risk of bias. We will then explore the impact of methodological heterogeneity through sensitivity analysis by analysing all studies together, including those with a high risk of bias, and comparing our primary analysis with our sensitivity analysis. If the results of the primary and sensitivity analyses differ, we will give precedence to the primary analysis because high risk of bias studies are known to be at risk of overestimating effect sizes. ${ }^{60}$

If the studies are heterogeneous (ie, if there is clinical, methodological and statistical heterogeneity), we will narratively summarise the characteristics and findings of all eligible studies according to the Synthesis Without Meta-analysis reporting guideline. ${ }^{61}$ To quantify the effectiveness of interventions, we will use the data provided in the studies to compute effect measures and 95\% CIs (ie, OR or relative risk for dichotomous outcomes, mean differences for continuous outcomes, hazard rate ratios 
for time-to-event outcomes and rates or rate ratios for count outcomes). ${ }^{62}$

We will assess the potential impact of reporting biases on the results of our review or meta-analysis by attempting to identify study protocols through the trials registry (WHO International Clinical Trials Registry Platform http://apps.who.int/trialsearch/), and through the use of funnel plots. After studies are stratified (table 3), outcomes that are reported in at least 10 studies will be assessed for publication bias by visually inspecting funnel plots for asymmetry. ${ }^{6364}$

We will interpret the quality of the evidence for each outcome according to the Grading Recommendations Assessment, Development and Evaluation system (GRADE). ${ }^{65}$ The quality of evidence ratings are very low (ie, the true effect is probably markedly different from the estimated effect), low (ie, the true effect might be markedly different from the estimated effect), moderate (ie, the true effect is probably close to the estimated effect) and high (highly confident that the true effect is similar to the estimated effect). Assessment of the quality of the evidence is determined by considering the risk of bias, inconsistency, indirectness, imprecision and publication bias. We will use established minimal clinically important differences to determine the clinical importance of effect sizes when possible. Similar to any meta-analysis we may conduct, we will give precedence to the primary analysis consisting of studies with low and unclear risk of bias.

\section{Economic synthesis}

We will report the main findings of economic studies, first stratified by high, low or unclear risk of bias. We will further stratify findings by study design (ie, cost-effectiveness, cost-utility, cost-benefit or cost-consequences). We will then stratify findings by type of intervention, outcome and cost measure.

To indicate whether an intervention might be judged favourably (or unfavourably) from an economic perspective, ${ }^{66}$ we will use the Dominance Ranking Matrix to classify the interventions into one of three options. ${ }^{67}$ Strong dominance for the intervention will be selected when the incremental cost-effectiveness measure shows the intervention as: (1) more effective and less costly than the comparator; or (2) effective and less costly; or (3) equal cost and more effective. In this case, from an efficiency perspective, decision makers should favour the intervention over the comparator (in circumstances similar to those of the evaluations). Weak dominance for the intervention will be selected when the measure shows the interventions as: (1) equally costly and effective as the comparator; or (2) more effective and more costly; or (3) less effective and less costly. In this case, no conclusion may be drawn about whether the intervention is preferable from an efficiency perspective without further information on the priorities or preferences of decision makers in a particular context. Decision makers must determine whether the cost/benefit trade-offs are worth the implemention of an intervention in their particular context. Lastly, non-dominance for the intervention will be selected when the measure shows the intervention as: (1) more costly and less effective; or (2) equally as costly and less effective; or (3) more costly and as effective. In this case, we will interpret the evidence as suggesting the comparator is favourable from an efficiency perspective (in circumstances similar to those of the evaluations).

\section{Qualitative synthesis}

We will stratify the qualitative findings similar to the quantitative and economic findings. We will first stratify the findings by risk of bias (ie, high/low/unclear), then by study approach or design (eg, qualitative descriptive, ethnography, grounded theory) and by intervention type and outcome.

Additionally, we will stratify findings according to individual perspective (ie, patient (children), caregivers (parents/guardians), healthcare providers, community service providers or others involved with the rehabilitation of back pain in children). We will use thematic synthesis to synthesise the qualitative research findings. ${ }^{68} 69$ First, we will enter all the text labelled as 'results' or 'findings' of the primary studies verbatim into EPPI-Reviewer. Then, pairs of trained reviewers will independently code each line of text according to its meaning and content, and group codes hierarchically into descriptive themes, including the a priori themes (intervention type and outcomes). Reviewers will also generate themes a posteriori to answer our review question (ie, experiences, preferences, expectations and valued outcomes regarding rehabilitation interventions for back pain in children). Reviewers will finalise the themes through discussion. We will give precedence to studies with low or unclear risk of bias. $^{70}$

Integration of quantitative, qualitative and economic evidence Various methods can be used to integrate diverse study types including: (1) juxtaposing findings in a matrix, (2) using logic models/conceptual framework, (3) analysing programme theory, (4) testing hypothesis derived using subgroup analysis and (5) qualitative comparative analysis. ${ }^{57}$ We will integrate the evidence by juxtaposing findings in a matrix to generate hypotheses regarding the effectiveness, cost-effectiveness and safety of rehabilitation interventions for back pain in children. We selected this methodology because it is suitable for comparing and contrasting the findings across the individual quantitative, qualitative and economic evidence syntheses in our review. ${ }^{57}$ The use of a matrix will allow us to explore heterogeneity in the findings of the quantitative studies and may indicate why some interventions may be effective, cost-effective and safe and some may not. ${ }^{57}$ For example, we may list themes from the qualitative synthesis along one side of the matrix, and then plot the interventions evaluated in the quantitative synthesis against the themes as either a match (when the intervention matched a theme) or a mismatch (when the intervention was the opposite of a theme) (as described in detail previously). ${ }^{18}$ We will also 
plot the economic evaluation findings against the corresponding intervention and theme. We will identify gaps in knowledge if a particular theme for an intervention does not match with any of the interventions evaluated in the quantitative studies.

\section{ETHICS AND DISSEMINATION}

Ethics approval is not required for this mixed studies review. Knowledge translation activities will include presentations to clinicians and researchers at national and international conferences; meetings with national and international decision makers (clinicians, health managers/administrators, policy makers and patients); publications in peer-reviewed journals; clinician and patient/caregiver resources; posts and lay language summaries on organisations' websites (open access) and other social media platforms.

\section{DISCUSSION}

Findings from this mixed studies review will advance our knowledge of the effectiveness, safety, user experience and cost-effectiveness of a wide range of rehabilitation interventions for children with back pain. This work will provide the evidentiary basis to develop clinical practice guidelines and care pathways outlining the evidencebased management of back pain in children, which can be adapted for specific settings (eg, hospitals, rehabilitation clinics and schools) and geographical regions. Specifically, decision makers should consider interventions that are identified as effective, safe, efficient and positively experienced by patients and caregivers. Mapping findings to the ICF framework will allow decision makers to use standardised language in the assessment and management of children during their care programme. This may further facilitate improvements in functioning and health outcomes in this patient population.

A potential limitation of our review is that our search strategy may miss potentially relevant studies, however, we have mitigated this by expanding our search strategy to include content experts and searching relevant websites. A potential risk is that there may be too little evidence available to answer our review questions.

Findings from this review will guide future research by identifying methodological limitations and knowledge gaps in the available literature. Future studies can be designed to address these limitations and gaps. This novel interpretation of quantitative, qualitative and economic evidence according to the ICF framework serves as a model for how outcomes related to functioning and health can be prioritised in future research.

\footnotetext{
Author affiliations

${ }^{1}$ Faculty of Health Sciences, Ontario Tech University, Oshawa, Ontario, Canada ${ }^{2}$ Centre for Disability Prevention and Rehabilitation, Ontario Tech University and Canadian Memorial Chiropractic College, Toronto, Ontario, Canada

${ }^{3}$ Epidemiology Division, Dalla Lana School of Public Health, University of Toronto, Toronto, Ontario, Canada
}

${ }^{4}$ Division of Graduate Studies, Canadian Memorial Chiropractic College, Toronto, Ontario, Canada

${ }^{5}$ EPPI-Centre, UCL Institute of Education, University College London, England, United Kingdom

${ }^{6}$ McMaster Midwifery Research Centre, McMaster University, Hamilton, Ontario, Canada

${ }^{7}$ Institute of Health Policy, Management and Evaluation, University of Toronto, Toronto, Ontario, Canada

${ }^{8}$ Department of Sport Science and Clinical Biomechanics, University of Southern Denmark, Odense, Denmark

${ }^{9}$ Nordic Institute of Chiropractic and Clinical Biomechanics, Odense, Denmark ${ }^{10}$ Division of General Medical Rehabilitation, Geneva University and University Hospitals, Geneva, Switzerland

${ }^{11}$ Division of Clinical Pharmacology and Toxicology, Multidisciplinary Pain Centre, Geneva University Hospitals, Geneva, Switzerland

${ }^{12}$ Department of Chiropractic, Faculty of Science and Engineering, Macquarie University, Sydney, New South Wales, Australia

${ }^{13}$ Rehabilitation Sciences, Faculty of Medicine, University of British Columbia, Vancouver, British Columbia, Canada

Acknowledgements This research is funded by the Canadian Chiropractic Research Foundation (grant number not applicable). The funder had no involvement in developing the protocol. The authors thank Marco Campello, Morgan Cark and Katie Pohlman for critically reviewing this manuscript. This research was undertaken, in part, thanks to funding from the Canada Research Chairs program to Pierre Côté who holds the Canada Research Chair in Disability Prevention and Rehabilitation at Ontario Tech University, and from the Canadian Chiropractic Research Foundation to Carol Cancelliere who holds a Research Chair in Knowledge Translation in the Faculty of Health Sciences at Ontario Tech University.

Contributors All authors (CC, JW, HY, SM, GB, HS, DR, LH, EP, CC, MS, GC, $\mathrm{LV}, \mathrm{AT}-\mathrm{V}$ and $\mathrm{PC}$ ) assisted in designing and planning the study, developing the research questions and systematic review methodology. CC and JW drafted the manuscript. All authors reviewed and revised the manuscript, and approved the final manuscript.

Funding This work was supported by the Canadian Chiropractic Research Foundation. The funding organisation was not involved in the design or conduct of this systematic review protocol.

Competing interests None declared.

Patient and public involvement Patients and/or the public were not involved in the design, or conduct, or reporting, or dissemination plans of this research.

Patient consent for publication Not required.

Provenance and peer review Not commissioned; externally peer reviewed.

Open access This is an open access article distributed in accordance with the Creative Commons Attribution Non Commercial (CC BY-NC 4.0) license, which permits others to distribute, remix, adapt, build upon this work non-commercially, and license their derivative works on different terms, provided the original work is properly cited, appropriate credit is given, any changes made indicated, and the use is non-commercial. See: http://creativecommons.org/licenses/by-nc/4.0/.

\section{ORCID iDs}

Carol Cancelliere http://orcid.org/0000-0003-1883-4970

Silvano Mior http://orcid.org/0000-0001-6575-2797

\section{REFERENCES}

1 Joergensen AC, Hestbaek L, Andersen PK, et al. Epidemiology of spinal pain in children: a study within the Danish national birth cohort. Eur J Pediatr 2019;178:695-706.

2 Aartun E, Hartvigsen J, Wedderkopp N, et al. Spinal pain in adolescents: prevalence, incidence, and course: a school-based two-year prospective cohort study in 1,300 Danes aged 11-13. BMC Musculoskelet Disord 2014;15:187.

3 Beynon AM, Hebert JJ, Lebouef-Yde C, et al. Potential risk factors and triggers for back pain in children and young adults. A scoping review, part I: incident and episodic back pain. Chiropr Man Therap 2019;27:58.

4 Kamper SJ, Yamato TP, Williams CM. The prevalence, risk factors, prognosis and treatment for back pain in children and adolescents: 
an overview of systematic reviews. Best Pract Res Clin Rheumatol 2016:30:1021-36

5 Calvo-Muñoz I, Gómez-Conesa A, Sánchez-Meca J. Prevalence of low back pain in children and adolescents: a meta-analysis. BMC Pediatr 2013;13:14.

6 King S, Chambers CT, Huguet A, et al. The epidemiology of chronic pain in children and adolescents revisited: a systematic review. Pain 2011:152:2729-38.

7 Jeffries LJ, Milanese SF, Grimmer-Somers KA. Epidemiology of adolescent spinal pain: a systematic overview of the research literature. Spine 2007;32:2630-7.

8 GBD 2017 Disease and Injury Incidence and Prevalence Collaborators. Global, regional, and national incidence, prevalence, and years lived with disability for 354 diseases and injuries for 195 countries and territories, 1990-2017: a systematic analysis for the global burden of disease study 2017. Lancet 2018;392:1789-858.

9 Batley S, Aartun E, Boyle E, et al. The association between psychological and social factors and spinal pain in adolescents. Eur $J$ Pediatr 2019;178:275-86.

10 Kamper SJ, Henschke N, Hestbaek L, et al. Musculoskeletal pain in children and adolescents. Braz J Phys Ther 2016;20:275-84.

11 Stallknecht SE, Strandberg-Larsen K, Hestbæk L, et al. Spinal pain and co-occurrence with stress and general well-being among young adolescents: a study within the Danish national birth cohort. Eur $J$ Pediatr 2017:176:807-14.

12 Dissing KB, Hestbæk L, Hartvigsen J, et al. Spinal pain in Danish school children - how often and how long? The CHAMPS Study-DK. BMC Musculoskelet Disord 2017:18:67.

13 Driehuis F, Hoogeboom TJ, Nijhuis-van der Sanden MWG, et al. Spinal manual therapy in infants, children and adolescents: a systematic review and meta-analysis on treatment indication, technique and outcomes. PLoS One 2019;14:e0218940.

14 Parnell Prevost C, Gleberzon B, Carleo B, et al. Manual therapy for the pediatric population: a systematic review. BMC Complement Altern Med 2019;19:60.

15 Michaleff ZA, Kamper SJ, Maher CG, et al. Low back pain in children and adolescents: a systematic review and meta-analysis evaluating the effectiveness of conservative interventions. Eur Spine J 2014;23:2046-58.

16 Harden A, Thomas J. Methodological issues in combining diverse study types in systematic reviews. Int J Soc Res Methodol 2005;8:257-71.

17 Moher D, Shamseer L, Clarke M, et al. Preferred reporting items for systematic review and meta-analysis protocols (PRISMA-P) 2015 statement. Syst Rev 2015;4:1

18 Cancelliere $\mathrm{C}$, Wong JJ, Yu H, et al. Postsurgical rehabilitation for adults with low back pain with or without radiculopathy who were treated surgically: protocol for a mixed studies systematic review. BMJ Open 2020;10:e036817.

19 National Institute for Health Research. International prospective register of systematic reviews (PROSPERO). Available: https://www. crd.york.ac.uk/prospero/ [Accessed 10 Jan 2019]

20 Moher D, Liberati A, Tetzlaff J, et al. Preferred reporting items for systematic reviews and meta-analyses: the PRISMA statement. $J$ Clin Epidemiol 2009;62:1006-12.

21 Tong A, Flemming K, Mclnnes E, et al. Enhancing transparency in reporting the synthesis of qualitative research: ENTREQ. BMC Med Res Methodol 2012;12:181.

22 World Health Organization. Definition of key terms. age groups and population. Available: https://www.who.int/hiv/pub/guidelines/ arv2013/intro/keyterms/en/ [Accessed 15 Jan 2020]

23 Amundsen PA, Evans DW, Rajendran D, et al. Inclusion and exclusion criteria used in non-specific low back pain trials: a review of randomised controlled trials published between 2006 and 2012. BMC Musculoskelet Disord 2018:19:113

24 North American Spine Society. NASS clinical guidelines: lumbar disc herniation with radiculopathy. Available: https://www.spine.org/ Documents/ResearchClinicalCare/Guidelines/LumbarDiscHerniation. pdf [Accessed 12 Dec 2018].

25 Merskey H, Bogduk N, et al, International Association for the Study of Pain. Classification of chronic pain: descriptions of chronic pain syndromes and definitions of pain terms. 2nd edn. Seattle: IASP Press, 1994.

26 The World Health Organization. World report on disability: chapter 4 rehabilitation. Available: https://www.spine.org/Documents/ ResearchClinicalCare/Guidelines/LumbarDiscHerniation.pdf

27 World Health Organization. The International classification of functioning, disability and health (ICF). Available: https://www.who. int/classifications/icf/icfbeginnersguide.pdf [Accessed 1 Dec 2018]

28 Fairbank JC, Couper J, Davies JB, et al. The Oswestry low back pain disability questionnaire. Physiotherapy 1980;66:271-3.
29 Roland M, Morris R. A study of the natural history of back pain. Part I: development of a reliable and sensitive measure of disability in lowback pain. Spine 1983;8:141-4.

30 McCormack HM, Horne DJ, Sheather S. Clinical applications of visual analogue scales: a critical review. Psychol Med 1988;18:1007-19.

31 Jensen MP, Turner JA, Romano JM, et al. Comparative reliability and validity of chronic pain intensity measures. Pain 1999;83:157-62.

32 Hicks CL, von Baeyer CL, Spafford PA, et al. The faces pain scalerevised: toward a common metric in pediatric pain measurement. Pain 2001:93:173-83.

33 Michaleff ZA, Kamper SJ, Stinson JN, et al. Measuring musculoskeletal pain in infants, children, and adolescents. J Orthop Sports Phys Ther 2017:47:712-30.

34 Chorpita BF, Yim L, Moffitt C, et al. Assessment of symptoms of DSM-IV anxiety and depression in children: a revised child anxiety and depression scale. Behav Res Ther 2000;38:835-55.

35 Spielberger CD. Manual for the state-trait anxiety inventory for children. Palo Alto: Consulting Psychologists Press, 1973.

36 Cieza A, Geyh S, Chatterji S, et al. Icf linking rules: an update based on lessons learned. J Rehabil Med 2005;37:212-8.

37 Ravens-Sieberer U, Gosch A, Rajmil L, et al. The KIDSCREEN-52 quality of life measure for children and adolescents: psychometric results from a cross-cultural survey in 13 European countries. Value Health 2008;11:645-58.

38 Varni JW, Seid M, Kurtin PS. PedsQL 4.0: reliability and validity of the pediatric quality of life inventory version 4.0 generic core scales in healthy and patient populations. Med Care 2001;39:800-12.

39 Health measures. Available: http://www.healthmeasures.net/exploremeasurement-systems/promis/intro-to-promis/list-of-pediatricmeasures [Accessed 14 Jan 2020].

40 Pohlman KA, O'Beirne M, Thiel $\mathrm{H}$, et al. Development and validation of providers' and patients' measurement instruments to evaluate adverse events after spinal manipulation therapy. Eur J Integr Med 2014;6:451-66

41 loannidis JPA, Evans SJW, Gøtzsche PC, et al. Better reporting of harms in randomized trials: an extension of the CONSORT statement. Ann Intern Med 2004;141:781-8.

42 Zorzela L, Boon H, Mior S, et al. Serious adverse events associated with pediatric complementary and alternative medicine. Eur J Integr Med 2014;6:467-72.

43 World Health Organization. Community-based rehabilitation. Available: https://www.who.int/disabilities/cbr/en/ [Accessed 1 Dec 2018].

44 Sampson M, McGowan J, Cogo E, et al. An evidence-based practice guideline for the peer review of electronic search strategies. J Clin Epidemiol 2009;62:944-52.

45 McGowan J, Sampson M, Salzwedel DM, et al. PRESS peer review of electronic search strategies: 2015 guideline statement. J Clin Epidemiol 2016;75:40-6.

46 Godin K, Stapleton J, Kirkpatrick SI, et al. Applying systematic review search methods to the grey literature: a case study examining guidelines for school-based breakfast programs in Canada. Syst Rev 2015;4:138.

47 Centre EfPaPlaC. EPPI-Reviewer 4 software. Available: eppi.ioe.ac. uk [Accessed 20 Nov 2019].

48 Belur J, Tompson L, Thornton A, et al. Interrater reliability in systematic review methodology: exploring variation in coder decision-making. Sociol Methods Res 2018:1-29.

49 Scottish Intercollegiate Guidelines Network (SIGN). Critical appraisal notes and checklists, 2019. Available: https://www.sign.ac.uk/ checklists-and-notes.html [Accessed 1 Feb 2019].

50 Lockwood C, Munn Z, Porritt K. Qualitative research synthesis: methodological guidance for systematic reviewers utilizing metaaggregation. Int J Evid Based Healthc 2015;13:179-87.

51 Pluye P, Gagnon M-P, Griffiths F, et al. A scoring system for appraising mixed methods research, and concomitantly appraising qualitative, quantitative and mixed methods primary studies in mixed studies reviews. Int J Nurs Stud 2009;46:529-46.

52 Drummond MF, Jefferson TO. Guidelines for authors and peer reviewers of economic submissions to the BMJ. The BMJ economic evaluation working party. BMJ 1996;313:275-83.

53 Higgins JPT, Savovic J, Page MJ, et al. Chapter 8: Assessing risk of bias in a randomized trial. In: Higgins JPT, Thomas J, Chandler $\mathrm{J}$, et al, eds. Cochrane Handbook for systematic reviews of interventions version 60. Cochrane, 2019.

54 Hoffmann TC, Glasziou PP, Boutron I, et al. Better reporting of interventions: template for intervention description and replication (TIDieR) checklist and guide. BMJ 2014;348:g1687. 
55 Booth A, Noyes J, Flemming K, et al. Formulating questions to explore complex interventions within qualitative evidence synthesis. BMJ Glob Health 2019;4:e001107.

56 Husereau D, Drummond M, Petrou S, et al. Consolidated health economic evaluation reporting standards (CHEERS) statement. BMJ 2013;346:f1049.

57 Harden A, Thomas J, Cargo M, et al. Cochrane qualitative and implementation methods group guidance series-paper 5: methods for integrating qualitative and implementation evidence within intervention effectiveness reviews. J Clin Epidemiol 2018;97:70-8.

58 Higgins JPT, Thompson SG. Quantifying heterogeneity in a metaanalysis. Stat Med 2002;21:1539-58.

59 Deeks JJ, Higgins JPT, Altman DG, et al. Chapter 10: Analysing data and undertaking meta-analyses. In: Higgins JPT, Thomas J, Chandler J, et al, eds. Cochrane Handbook for systematic reviews of interventions version 60. Cochrane, 2019.

60 Higgins JPT, Altman DG, Gøtzsche PC, et al. The Cochrane collaboration's tool for assessing risk of bias in randomised trials. BMJ 2011;343:d5928.

61 Campbell M, McKenzie JE, Sowden A, et al. Synthesis without metaanalysis (SWiM) in systematic reviews: reporting guideline. BMJ 2020;368:16890.

62 McKenzie JE, Brennan SE. Chapter 12: Synthesizing and presenting findings using other methods. In: Higgins JPT TJ, Chandler J, Cumpston M, et al, eds. Cochrane Handbook for systematic reviews of interventions version 60. Cochrane, 2019.

63 Page MJ, Higgins JPT, Sterne JAC. Chapter 13. Assessing risk of bias due to missing results in a synthesis. In: Higgins JPT, Thomas
$\mathrm{J}$, Chandler J, et al, eds. Handbook for systematic reviews of interventions version 60. Cochrane, 2019.

64 Egger M, Davey Smith G, Schneider M, et al. Bias in meta-analysis detected by a simple, graphical test. BMJ 1997;315:629-34.

65 Schünemann HJ, Higgins JP, Vist GE, et al. Chapter 14: Completing 'Summary of findings' tables and grading the certainty of the evidence. In: Higgins JPT TJ, Chandler J, Cumpston M, et al, eds. Cochrane Handbook for systematic reviews of interventions version 60. Cochrane, 2019.

66 Shemilt I, Aluko P, Graybill E, et al. Economic evidence. In: Higgins JPT TJ, Chandler J, Cumpston M, et al, eds. Cochrane Handbook for systematic reviews of interventions version 60, 2019.

67 Joanna Briggs Institute. Chapter 6: Systematic reviews of economic evidence, 2019. Available: https://wiki.joannabriggs.org/display/ MANUAL/Chapter+6\%3A+Systematic+reviews+of+economic+ evidence [Accessed 1 Feb 2020].

68 Thomas J, Harden A. Methods for the thematic synthesis of qualitative research in systematic reviews. BMC Med Res Methodol 2008;8:45.

69 Noyes J, Booth A, Flemming K, et al. Cochrane qualitative and implementation methods group guidance series-paper 3: methods for assessing methodological limitations, data extraction and synthesis, and confidence in synthesized qualitative findings. $J$ Clin Epidemiol 2018;97:49-58.

70 Slavin RE. Best evidence synthesis: an intelligent alternative to metaanalysis. J Clin Epidemiol 1995;48:9-18. 